Mario Rojas Delgado

Eduardo López Sandoval

Silvia Zaldívar Peña

\title{
Desalación del agua de mar para uso industrial
}

\section{INTRODUCCIÓN}

El agua se está convirtiendo en un bien escaso, debido a que su consumo se incrementa incesantemente, como resultado del crecimiento de la población y la mejora de su nivel de vida. Asimismo, los expertos prevén para el año 2015 el agotamiento de los recursos del líquido indispensable consumibles en las regiones habitables del planeta; sin embargo, los continentes están rodeados de agua salada. De otra parte, los cambios climáticos producidos por la contaminación del medio ambiente y la desertificación están alterando los sistemas hídricos en diversas partes del mundo, generando escasez de agua tanto para el consumo humano como para la agricultura.

Frente a este grave problema, numerosos estudiosos se han planteado la posibilidad de eliminar las sales del agua de los océanos mediante un proceso barato, lo que podría resolver las necesidades de agua más urgentes de la humanidad. De esta manera, teniendo en cuenta que el área de cultivo equivale solo a una décima parte de la superficie de los continentes, las tierras áridas, que constituyen más de la tercera parte de esta, podrían ser fértiles otra vez (ILCE, 1985).

Desde hace décadas se dispone de las tecnologías necesarias para desalar el agua a gran escala, las cuales demandan una inversión muy elevada. Sin embargo, estas se han ido perfeccionando progre- 
sivamente, con el fin de obtener costos de procesamiento competitivos frente a otros sistemas de suministros de agua.

Además de sus elevados costos, el proceso de desalinización del agua enfrenta dificultades medioambientales, debido a que tras la obtención del agua dulce se genera un residuo que es la salmuera (agua con alto contenido de sal), cuyo manejo y disposición puede convertirse en un problema ecológico (Universidad de Alicante, 2003).

El agua es un recurso renovable, por ser la consecuencia de la actuación de la energía solar sobre el planeta, la cual produce la evaporación de los océanos, distribuyéndola alrededor de la Tierra; pero el procesamiento y el suministro de agua para una gran parte de la población demandan también un costo muy alto.

De acuerdo con cifras recientes, por lo menos 34 países de Asia, África y del Medio Oriente se encuentran clasificados como "hidroestresados", cuyas fuentes renovables de agua están por debajo de los $1.700 \mathrm{~m}^{3}$ per cápita, y las proyecciones al 2025 indican que el número de habitantes en esos países aumentará a 3.000 millones. En la actualidad 470 millones de habitantes viven en zonas con severa escasez de agua, entre las que se incluyen el norte de China, el norte de África, el Medio Oriente, el norte de la India, parte de México y el oeste de Estados Unidos (Unesco, 2000).

En el Perú existen 1,8 millones de hectáreas de tierras aptas para el riego, de las cuales se están regando 1,1 millones de hectáreas, quedando 700 mil hectáreas aún sin suministro de agua dulce. Asimismo, en la costa peruana, con aproximadamente 1,2 millones de hectáreas aptas para el riego, solo 736 mil hectáreas están irrigadas (61\%). Además, existen áreas afectadas por el deficiente drenaje y la creciente salinidad, producto del mal manejo de las aguas de riego y de los suelos.

En esta zona el problema más severo es la degradación de los suelos por efectos de la salinización de las tierras. El clima árido de la región, la naturaleza salina de algunos suelos, el deficiente sistema de drenaje, la mala práctica del manejo de agua y el cultivo del arroz (en la costa norte) son las causas principales de este problema, que abarca 306.700 hectáreas (Inrena, 2004; Minag, 2003). 


\section{TECNOLOGÍAS DISPONIBLES}

\subsection{Métodos de desalación}

El proceso de desalación consiste en separar el agua de las sales; por lo tanto, la primera clasificación de los métodos de desalación es la que se refiere a la forma de separación de las sales y el agua. Las siguientes clasificaciones se hacen según el tipo de energía utilizada para el proceso, y, finalmente, por el proceso físico de la desalación.

A continuación se mencionan los principales procesos de desalación de agua del mar existentes, con sus correspondientes denominaciones y siglas en inglés:

\subsubsection{Principales procesos comerciales}

- Procesos por destilación (evaporación-condensación) (Miller, 2003)

- Evaporación rápida con múltiples etapas (MSF: Multi-Stage Flash).

- Evaporación con múltiples efectos (MEE o MED: Multi-Effect Evaporation).

- Compresión térmica de vapor (TVC: Thermal Vapor Compression)

- Compresión mecánica de vapor (MVC: Mechanical Vapor Compression).

- Procesos por membrana

- Ósmosis inversa (RO: Reverse Osmosis).

- Electrodiálisis (ED: Electrodialysis).

Procesos alternativos (Moss et al., 1994)

- Procesos por cristalización

- Desalación por congelamiento (FD: Freeze Desalting).

- Formación de hidratos gaseosos (GHP: Gas Hydrate Process).

- Procesos por humidificación

- Proceso evaporativo condensante (DEP: Dew-Evaporation Process).

- Invernadero marino (SG: Seawater Greenhouse).

- Destilación por membrana (ME: Membrane Distillation).

- Evaporación mecánicamente intensificada (MIE: Mechanically Intensified Evaporation).

- Condensación atmosférica de agua (AWC: Atmospheric Water Condensing).

- Procesos accionados por efectos marinos 
- Bombeo osmótico (OP: Osmotic Pumping).

- Bombeo accionado por ondas marinas (WP: Wave Pumping).

- Procesos accionados por golpe de ariete (WP: Waterhammer Process).

- Proceso "pato cabeceador" (NDP: Nodding Duck Process).

- Procesos solares

- Destilador solar (SS: Solar Still).

\subsubsection{Otros procesos}

- Intercambio iónico (IE: Ion Exchange).

- Flujo transversal capacitivo (FTC: Flow Through Capacitor).

- Extracción líquido-líquido (LLE: Liquid-Liquid Extraction).

- Ósmosis inversa centrífuga (CRO: Centrifugal Reverse Osmosis).

- Compresión rotatoria de vapor (RVC: Rotary Vapor Compression).

- Desionización capacitiva (CDT: Capacitive Deoionization Technology; CDI: Capacitive Deionization).

- Procesos MSF/MEE con turbina.

- Procesos MSF/MEE con cogeneración.

- Procesos duales (DP).

- Procesos híbridos (HP).

El cuadro 1 muestra la clasificación de los métodos existentes.

\section{Cuadro 1}

Métodos de desalación existentes en el mercado

\begin{tabular}{|c|c|c|c|}
\hline Material separado & Energía & Proceso & Método \\
\hline \multirow{5}{*}{ Agua de sales } & \multirow{3}{*}{ Térmica } & Evaporación & $\begin{array}{l}\text { Destilación rápida (MSF) } \\
\text { Destilación multiefecto (MEE) } \\
\text { Termocompresión de vapor (TVC) } \\
\text { Destilación solar (SS) }\end{array}$ \\
\hline & & Cristalización & $\begin{array}{l}\text { Congelación (FD) } \\
\text { Formación de hidratos (GHP) }\end{array}$ \\
\hline & & Filtración y evaporación & Destilación con membranas (ME) \\
\hline & \multirow{2}{*}{ Mecánica } & Evaporación & Compresión mecánica vapor (MVC) \\
\hline & & Filtración & Ósmosis Inversa (RO) \\
\hline \multirow{2}{*}{ Sales de agua } & Eléctrica & Filtración selectiva & Electrodiálisis (ED) \\
\hline & Química & Intercambio & Intercambio iónico (IE) \\
\hline Sales de agua & Eléctrica & Desionización & Desionización capacitiva CDT(CDI) \\
\hline
\end{tabular}

Fuente: Valero, 2000.

Elaboración propia. 


\subsection{Valoración de diferentes características deseables para los métodos de desalación existentes en el mercado}

A modo de síntesis, es conveniente realizar un análisis comparativo de las ventajas e inconvenientes de cada uno de estos métodos tecnológicamente avanzados para la producción de agua desalada. El cuadro 2 muestra la valoración de los métodos existentes frente a ciertas características exigibles para un método desalador.

\section{Cuadro 2}

Valoración de diferentes características deseables para los métodos de desalación

\begin{tabular}{|l|c|c|c|c|c|c|}
\hline \multicolumn{1}{|c|}{ Características } & MSF & MEE-TVC & VC & RO & ED & CDT(CDI) \\
\hline Tipo de energía & térmica & térmica & eléctrica & eléctrica & eléctrica & eléctrica \\
\hline Consumo energético & $\begin{array}{c}\text { Alto } \\
(>200)\end{array}$ & $\begin{array}{c}\text { alto/medio } \\
(150-200)\end{array}$ & $\begin{array}{c}\text { medio } \\
(100-150)\end{array}$ & $\begin{array}{c}\text { bajo } \\
(<80)\end{array}$ & $\begin{array}{c}\text { bajo } \\
(<30)\end{array}$ & $\begin{array}{c}\text { bajo } \\
(<50)\end{array}$ \\
\hline Costo de instalaciones & Alto & alto/medio & alto & medio & medio & medio \\
\hline $\begin{array}{l}\text { Capacidad de producción } \\
(m 3 / \text { día })\end{array}$ & $\begin{array}{c}\text { Alta } \\
(>50.000)\end{array}$ & $\begin{array}{c}\text { media } \\
(<20.000)\end{array}$ & $\begin{array}{c}\text { baja } \\
(<5.000)\end{array}$ & $\begin{array}{c}\text { alta } \\
(>50.000)\end{array}$ & $\begin{array}{c}\text { media } \\
(<30.000)\end{array}$ & $\begin{array}{c}\text { Media } \\
(<50.000)\end{array}$ \\
\hline Posibilidad de ampliación & difícil & difícil & difícil & fácil & fácil & fácil \\
\hline Fiabilidad de la operación & Alta & media & baja & alta & alta & alta \\
\hline $\begin{array}{l}\text { Desalación del agua } \\
\text { Calidad del agua desalada } \\
\text { (ppm) }\end{array}$ & $\begin{array}{c}\text { Alta } \\
(<50)\end{array}$ & $\begin{array}{c}\text { alta } \\
(<50)\end{array}$ & $\begin{array}{c}\text { alta } \\
(<50)\end{array}$ & $\begin{array}{c}\text { media } \\
(300-500)\end{array}$ & $\begin{array}{c}\text { media } \\
(<300)\end{array}$ & $\begin{array}{c}\text { media } \\
(300-500)\end{array}$ \\
\hline $\begin{array}{l}\text { Superficie de terreno requeri- } \\
\text { da para instalación }\end{array}$ & mucha & media & poca & poca & poca & poca \\
\hline
\end{tabular}

Fuente: Valero, 2000.

Elaboración propia.

\section{CALIDAD DE LAS AGUAS}

Los procesos de destilación consumen la misma cantidad de energía independientemente de la salinidad del agua aportada, por lo que solo son apropiadas para la desalación de aguas marinas. Las aguas especialmente puras para aplicaciones industriales específicas necesitan determinados procesos, como el intercambio iónico, o postratamientos si se utiliza inicialmente un proceso de ósmosis inversa.

Si se toma como referencia la cantidad de sólidos totales disueltos, el cuadro 3 muestra la denominación del agua en función de su salinidad. 


\section{Cuadro 3}

Rangos de salinidad de los diferentes tipos de agua

\begin{tabular}{lc}
\hline Denominación del agua & Salinidad $(\mathrm{ppm}$ de TDS) \\
\hline Ultrapura & 0,03 \\
Pura (calderas) & 0,3 \\
Desionizada & 3 \\
Dulce (potable) & $<1.000$ \\
Salobre & $1.000-10.000$ \\
Salina & $10.000-30.000$ \\
Marina & $30.000-50.000$ \\
Salmuera & $>50.000$ \\
\hline
\end{tabular}

* TDS = Total de sólidos disueltos ppm $=$ partes por millón

Fuente: Fariñas, 1999; Valero, 2001.

Elaboración propia.

En cuanto a la salinidad de los mares y océanos del planeta, esta no es constante, ni mucho menos, como se aprecia en el cuadro 4.

\begin{tabular}{lc} 
Cuadro $\begin{array}{c}\text { C } \\
\text { Salinidad media de los mares } \mathbf{y} \text { océanos }\end{array}$ \\
\hline Mar/océano & Salinidad (ppm de TDS) ${ }^{*}$ \\
\hline Mar Báltico & 28.000 \\
Mar del Norte & 34.000 \\
Océno Pacífico & 33.600 \\
Océno Atlántico Sur & 35.000 \\
Mar Mediterráneo & 36.000 \\
Mar Rojo & 44.000 \\
Golfo Pérsico & $43.000-53.000$ \\
Mar Muerto & $50.000-80.000$ \\
Media Mundial & 34.800 \\
\hline
\end{tabular}

Fuente: Valero, 2001.

Elaboración propia. 


\subsection{Proceso desalador aplicable en función del agua cruda}

El agua pura no conduce la electricidad, pero se va convirtiendo en conductora con la adición de diversos constituyentes de naturaleza electrolítica, así como de otros elementos químicos que contiene el agua. Esto ocurre especialmente cuando se trata de aguas salobres o residuales, que incluso pueden contener componentes de difícil eliminación. Lo descrito, a lo que se añade la temperatura del agua, influyen mucho al momento de elegir el proceso desalador. Por ejemplo, la dureza y temperatura de las aguas del Golfo Pérsico siempre han condicionado fuertemente el uso de membranas de ósmosis inversa hasta hace muy pocos años. El pretratamiento de esta agua cruda, necesario para cada proceso desalador es a veces más costoso y complejo que el proceso desalador en sí, por lo que aunque un pretratamiento pueda suplir las deficiencias que plantea ese proceso su costo y complejidad pueden finalmente hacer inviable esa instalación. El cuadro 5 resume el tipo de proceso básico que se puede utilizar en función del tipo de agua de aporte especificada.

\section{Cuadro 5}

Proceso desalador aplicable en función del tipo de agua cruda

\begin{tabular}{lcc}
\hline \multicolumn{1}{c}{ Proceso } & Agua de mar & Agua salobre \\
\hline MSF & Aplicable & - \\
MEE & Aplicable & - \\
TVC & Aplicable & - \\
VC & Aplicable & - \\
RO & Aplicable & Aplicable \\
ED & - & Aplicable \\
CDT(CDI) & En desarrollo & Aplicable \\
\hline
\end{tabular}

Fuente: Valero, 2001.

Elaboración propia.

\subsection{Calidad de agua requerida}

Para ciertos procesos industriales pueden usarse aguas con hasta $5.000 \mathrm{ppm}$, pero en otros, como en las centrales eléctricas, el límite máximo es 0,3 ppm. En la agricultura, algunos cultivos toleran hasta 2.000 ppm, aunque ello depende de la tierra, el clima, la composición del agua salobre, el método de riego y los fertilizantes aplicados. En 
cuanto al consumo humano, su límite es de 1.000 ppm, aunque en climas excesivamente cálidos un aporte extra de sales (principalmente cloruro sódico) puede ser beneficioso para el cuerpo humano.

\section{DESCRIPCIÓN DE LAS PRINCIPALES PLANTAS DESALADORAS}

\subsection{Plantas desaladoras MSF}

Las plantas MSF comprenden procesos térmicos de evaporación rápida (flash) y condensación de agua (también denominados procesos de destilación flash). Las etapas de este proceso se han diseñado de tal modo que permiten que el calor latente de evaporación se recupere para precalentar el flujo de agua de entrada al sistema tal como se muestra en la figura 1.

Las plantas MSF se han usado ampliamente en el Medio Oriente y representan un $40 \%$ de la capacidad de desalación instalada a escala mundial. Para maximizar la recuperación del agua cada etapa de una planta MSF opera a menores presiones de modo sucesivo.

Figura 1

Planta de evaporación rápida con múltiples etapas

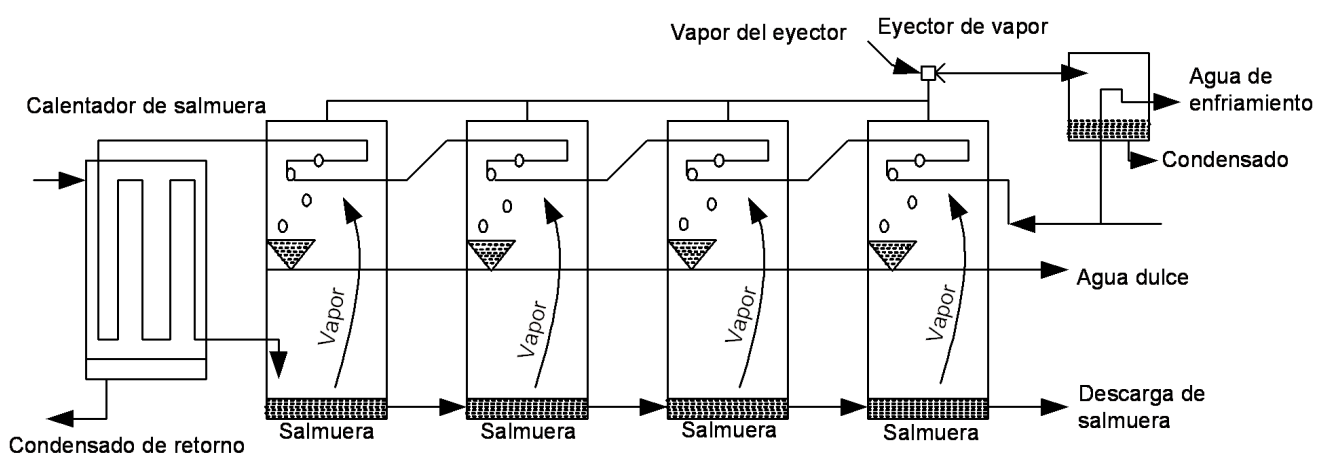

Fuente: Miller, 2003.

Elaboración propia. 


\subsection{Plantas desaladoras MEE (MED)}

Las plantas MEE comprenden los procesos relacionados con los procesos de evaporación de una planta MSF, que se desarrollaron en la década de 1950. Sin embargo, por problemas de incrustación de sales en los tubos de los intercambiadores de calor se han ido remplazando por plantas MSF. Las plantas MEE no son tan usadas pero llaman la atención por tener mejor rendimiento que las plantas MSF. Véase el esquema del proceso en la figura 2.

Figura 2

Planta de evaporación con múltiples efectos

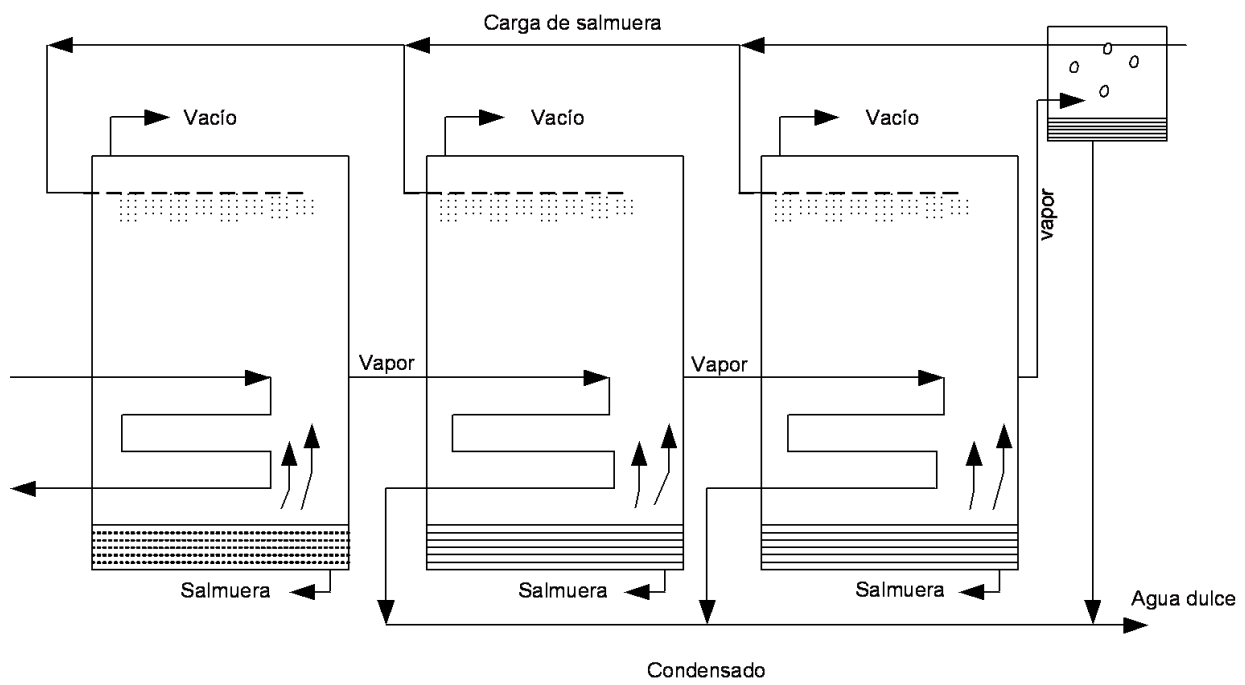

Fuente: Miller, 2003.

Elaboración propia.

\subsection{Plantas desaladoras MVC (TVC)}

Las plantas desaladoras con procesos de compresión de vapor se basan en operaciones con presión reducida para lograr la evaporación, tal como se muestran en la figura 3 . El calor de evaporación es suministrado por la compresión de vapor, sea por compresión mecánica (MVC) o por eyección de vapor (TVC). Estas plantas son recomendables para instalaciones pequeñas o medianas (de 3.000 a $20.000 \mathrm{~m}^{3} /$ día). 
Figura 3

Planta de compresión mecánica de vapor

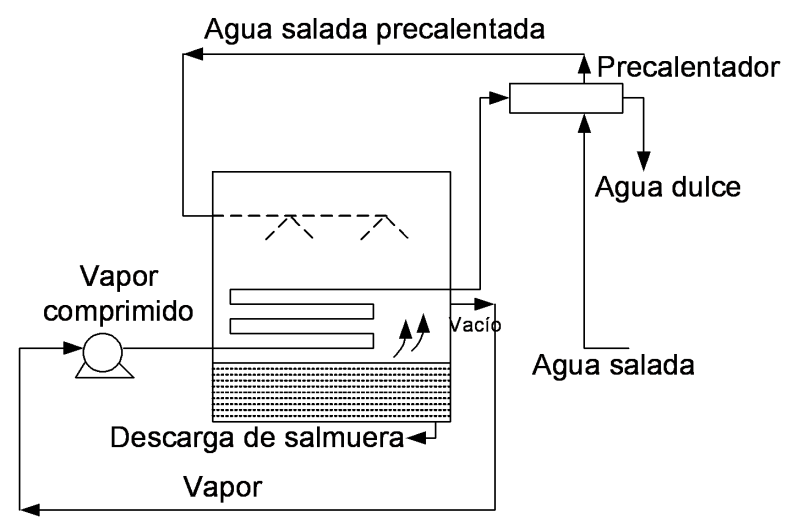

Fuente: Miller, 2003.

Elaboración propia.

\subsection{Plantas desaladoras RO}

Las plantas por ósmosis inversa (RO) emplean procesos de separación por membrana, que permiten recuperar el agua a partir de una solución salina presurizada a un punto tal que supere la presión osmótica de la solución (véase figura 4). Por su amplio rango de capacidades de operación son muy empleadas en Europa, Norteamérica y Sudamérica.

\section{Figura 4}

Planta de ósmosis inversa

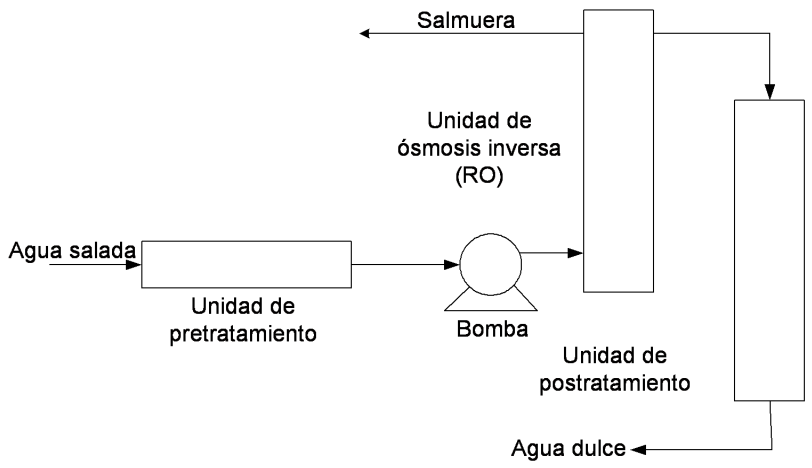

Fuente: Miller, 2003.

Elaboración propia. 


\subsection{Planta desaladora CDT (CDI)}

El corazón de un sistema de desionización capacitiva CDT (CDI) es un conjunto de capas formadas por mallas de fibras de carbono que sirven como electrodos. Las mallas estratificadas están dispuestas de manera que al aplicarse corriente continua la polaridad de las capas se alternan. Las sales disueltas en agua son atraídas por las capas de polaridad opuesta, removiendo así las sales disueltas desde el agua. Cuando hay suficientes sales depositadas en los electrodos, de tal forma que la conductividad se incrementa por encima del punto de consigna fijado, los electrodos son regenerados haciendo tierra (véase figura 5).

Figura 5

Fundamento del proceso purificador de agua CDT (CDI)

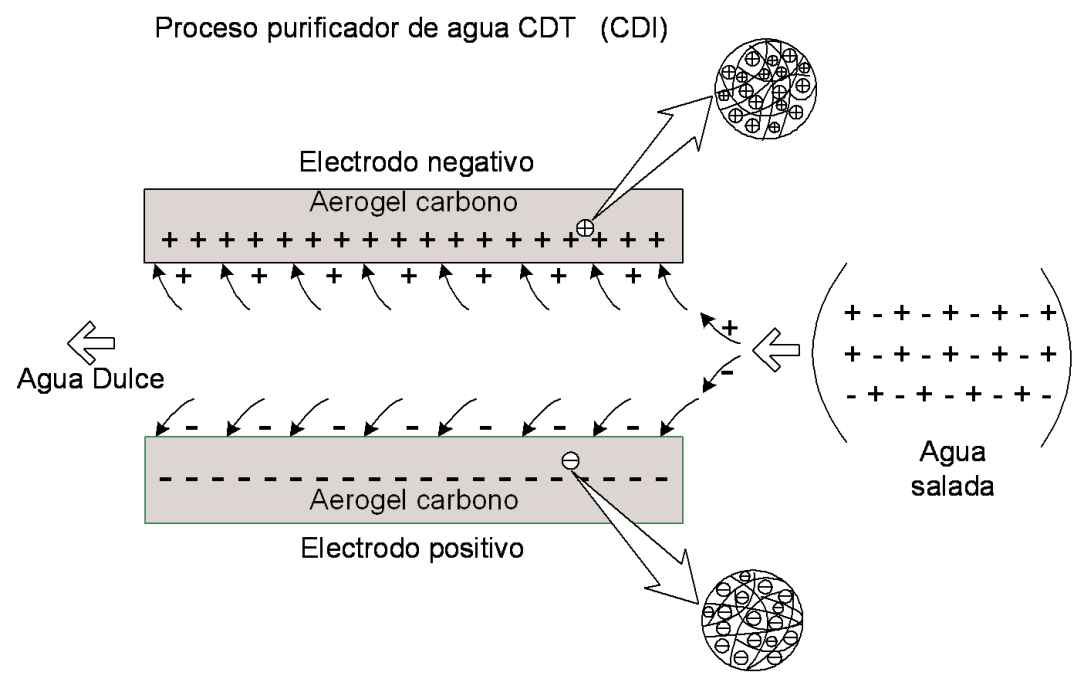

Fuente: CDT Systems Inc., 2004; LLNL, 2003.

Esencialmente los contaminantes se desprenden del electrodo y son desaguados hacia fuera del sistema. La descarga tiene entre 5 a 10 veces la concentración original y pasa a través de una válvula hacia el desagüe. Terminada la descarga, la polaridad se revierte y empieza un nuevo ciclo de operación.

La principal ventaja que tiene el proceso de desionización capacitiva (CDT) respecto del proceso de ósmosis inversa (RO) es que el pretrata- 
miento no es un asunto de mayor importancia. Los factores que afectan las membranas - sólidos suspendidos, cloro y productos orgánicos- no afectan los electrodos. Un indicativo del interés en esta tecnología es la opinión de los investigadores del U.S. Bureau of Reclamation que consideran que el proceso CDT es uno de los más prometedores desarrollos en el campo de la desalinización de agua de los últimos veinte años.

\section{DESCRIPCIÓN DE PLANTAS ELÉCTRICAS PARA COGENERACIÓN CON PLANTAS DESALADORAS}

\subsection{Ciclo simple con turbina de gas (SCGT)}

Una planta de ciclo simple con turbina de gas comprende el quemado de un combustible (quemador) con aire comprimido (compresor), lo que produce gases de combustión a alta temperatura que accionan una turbina de gas para generar corriente eléctrica (generador eléctrico). En la figura 6 se presenta el diagrama del ciclo simple con ciertos detalles de las unidades y de modo simplificado, obsérvese que los gases de salida de la turbina de gas son arrojados al entorno y se consigue entre $35 \%$ y $38 \%$ de eficiencia térmica.

\section{Figura 6}

\section{Ciclo simple con turbina de gas}

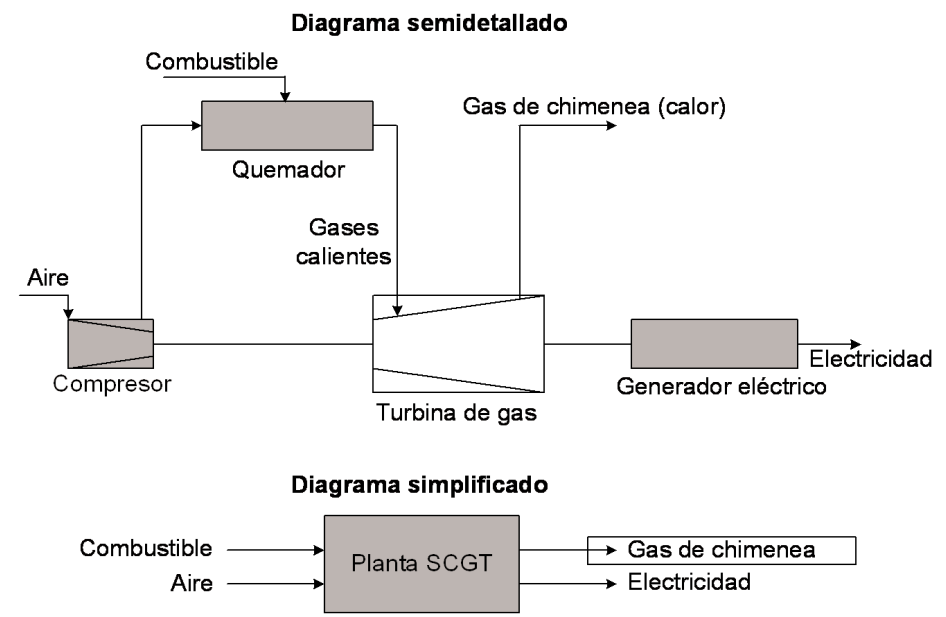

Fuente: Minem, 2003.

Elaboración propia. 


\subsection{Ciclo combinado con turbinas de gas y de vapor (CCGST)}

Una planta de ciclo combinado con turbina de gas y turbina de vapor comprende un ciclo simple con turbina de gas, del cual se aprovecha el calor residual de los gases de chimenea con un cierto efecto de calentamiento suplementario para activar una caldera que produce vapor para accionar una turbina de vapor, que finalmente genera un segundo efecto de corriente eléctrica. En la figura 7 se muestra el diagrama del ciclo combinado con ciertos detalles de las unidades y de modo simplificado, obsérvese que hay dos efectos de generación de electricidad y puede tenerse la posibilidad de exportar el calor residual de los gases de combustión y de vapor residual o condensado.

Figura 7

Ciclo combinado con turbinas de gas y de vapor
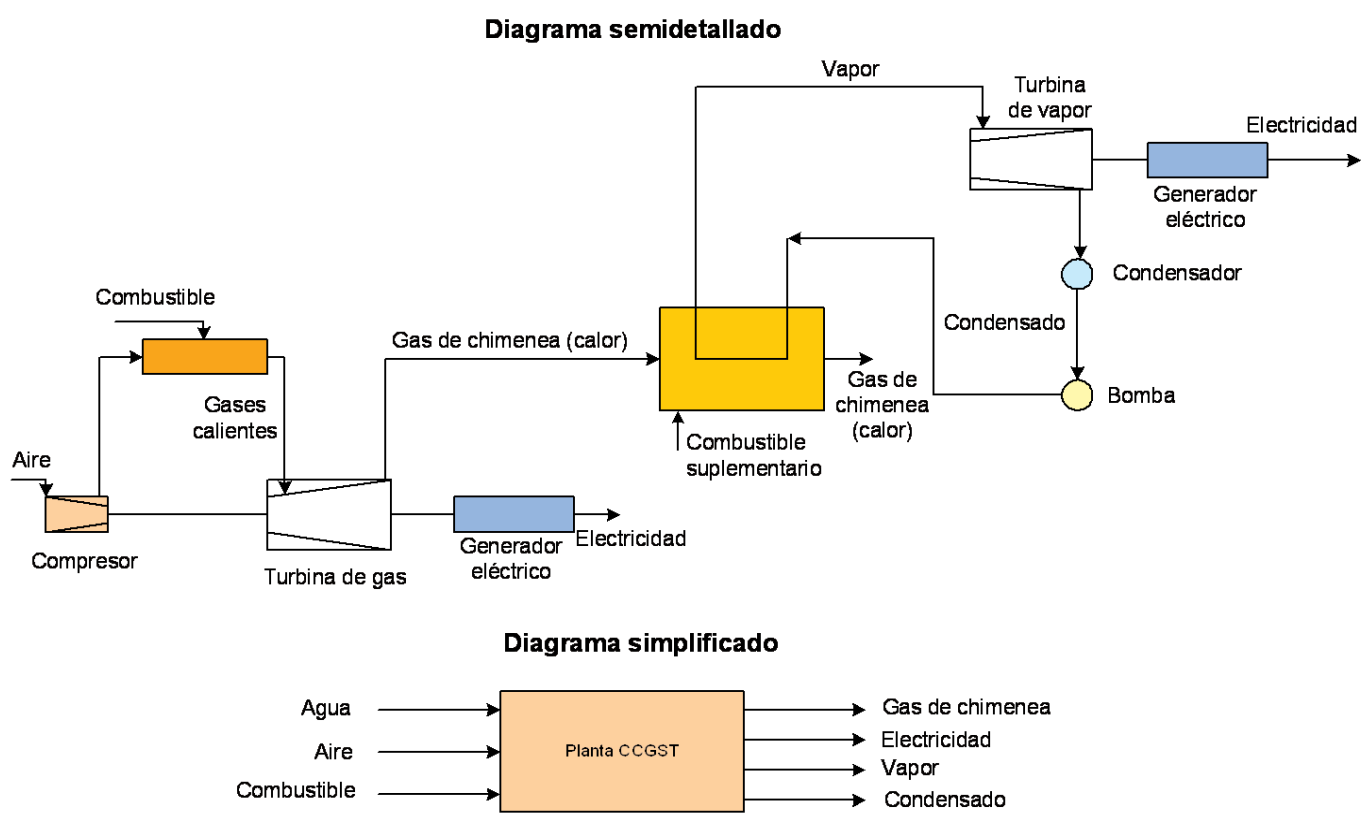

Fuente: Minem, 2003.

Elaboración propia. 


\subsection{Tecnologías duales de ciclos combinados (CCGST) con plantas desaladoras}

La tecnología dual combina una planta desaladora con una planta de potencia (en estos casos con ciclo de potencia combinado) con el propósito de optimizar el uso y exportación de calor residual, vapor residual y corriente eléctrica.

En las figuras 8 y 9 se muestran dos tipos de plantas desaladoras duales, ambas conectadas a una planta de ciclos combinados (CCGST). La primera es una planta de evaporación rápida con múltiples etapas (MSF) y la segunda es una planta de evaporación con múltiples efectos (MEE).

Figura 8

Planta dual CCGST- MSF

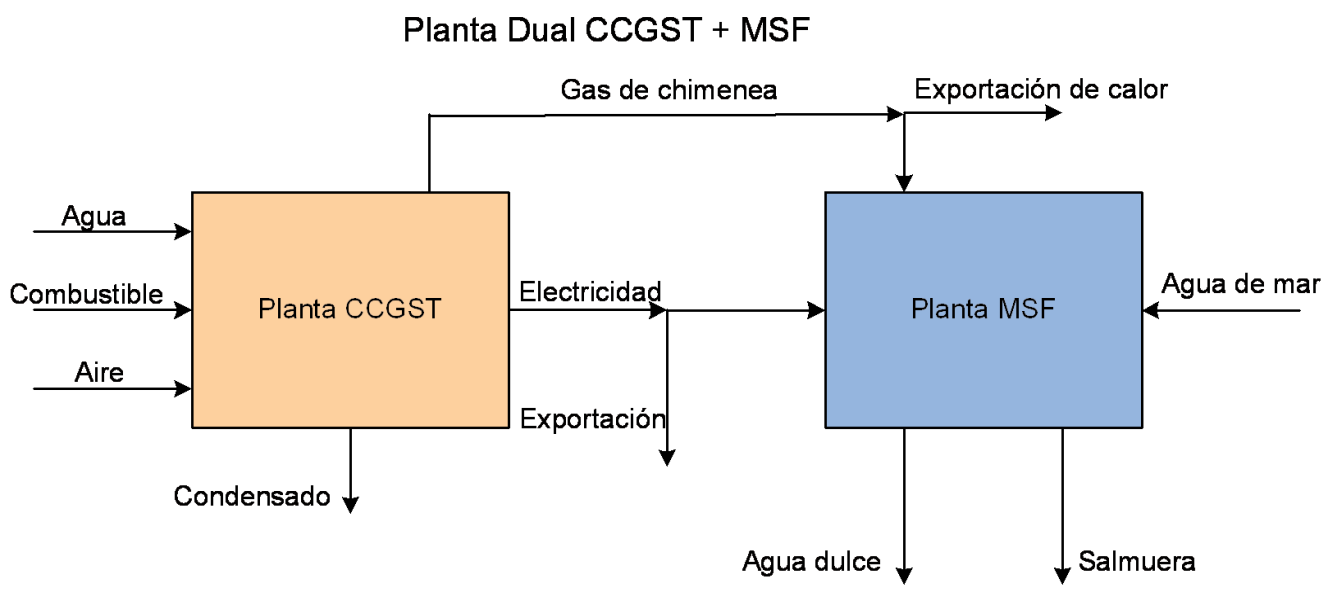

Fuente: Ettouney et al., 2002; Valero, 2001.

Elaboración propia. 
Figura 9

Planta dual CCGST- MEE

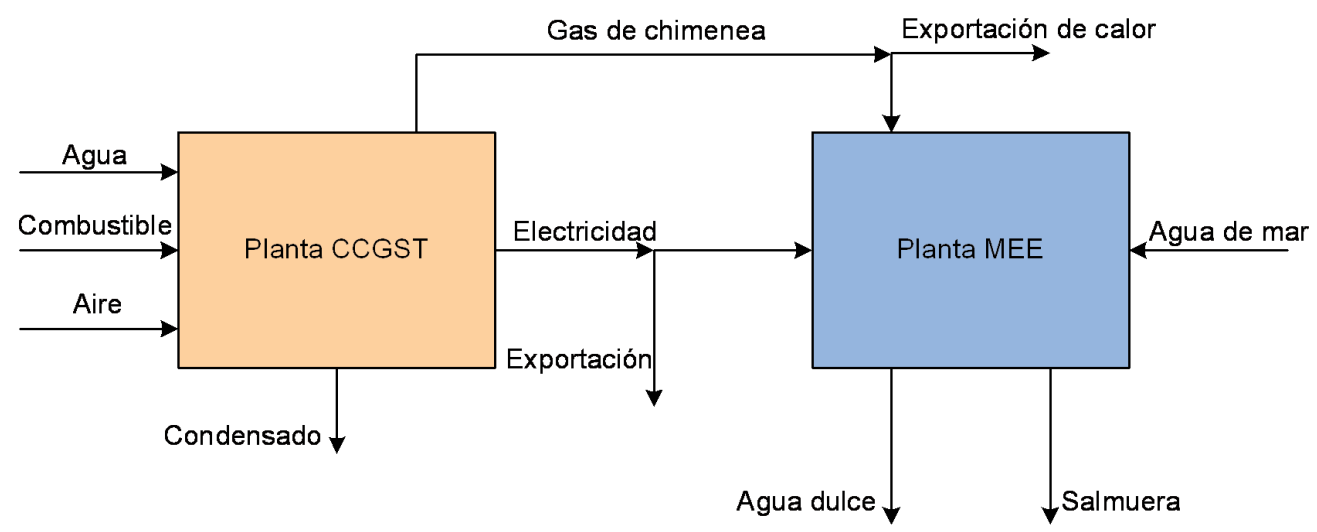

Fuente: Ettouney et al., 2002; Valero, 2001.

Elaboración propia.

\section{TECNOLOGÍAS RECOMENDABLES}

\subsection{Diseño conceptual de tecnologías desaladoras seleccionadas}

En las figuras 10, 11 y 12, que se muestran a continuación, se dan los principales detalles del diseño conceptual de tres plantas desaladoras analizados por los autores de este trabajo. Cabe destacar que la tecnología dual de ciclo combinado de potencia con turbina de gas y de vapor (CCGST) y desionización capacitiva (CDT) es responsabilidad de los autores de este artículo (figura 11); los otros dos casos son resultado de una adaptación de las recomendaciones proporcionadas por especialistas de España, Estados Unidos y Kuwait. 
6.2 Tecnología dual de ciclo combinado de potencia con turbinas de gas y de vapor (CCGST) y ósmosis inversa (RO)

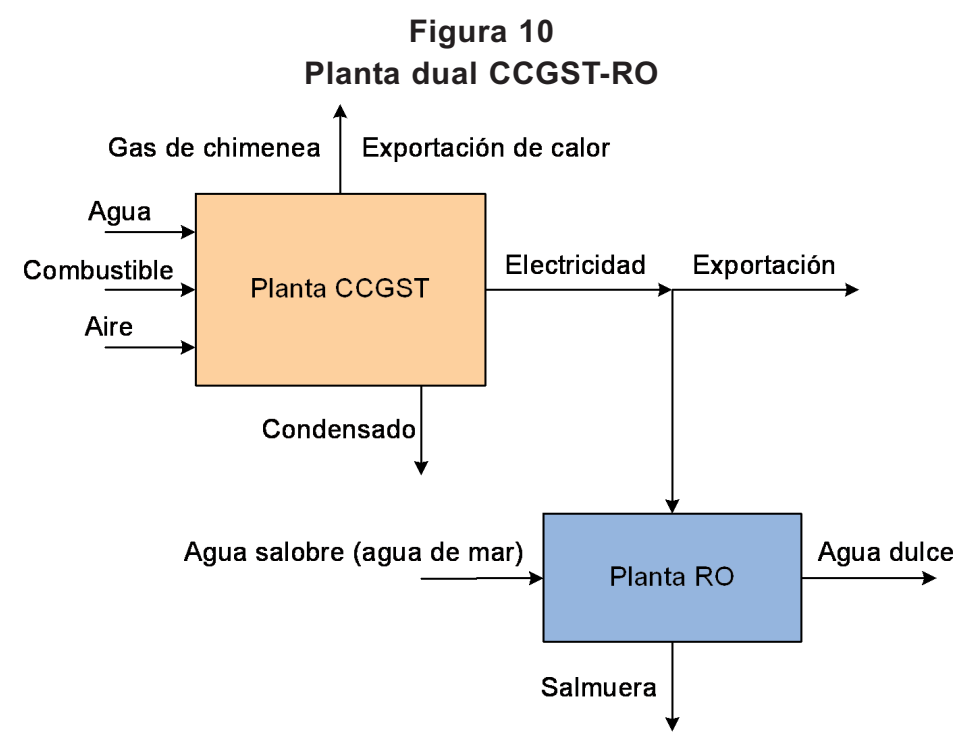

Fuente: Ettouney et al., 2002; Valero, 2001.

Elaboración propia.

6.3 Tecnología dual de ciclo combinado de potencia con turbina de gas y de vapor (CCGST) y desionización capacitiva (CDT)

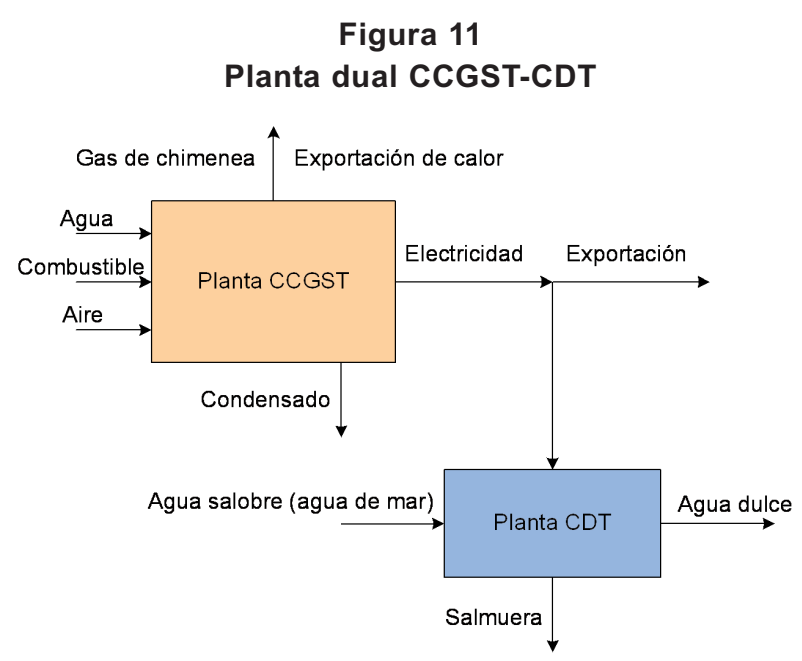

Fuente y elaboración propias. 


\subsection{Tecnología híbrida de ciclo combinado de potencia con turbina de gas y de vapor (CCGST), ósmosis inversa (RO) y evaporación con múltiples efectos (MEE)}

Figura 12

\section{Planta híbrida CCGST-RO-MEE}

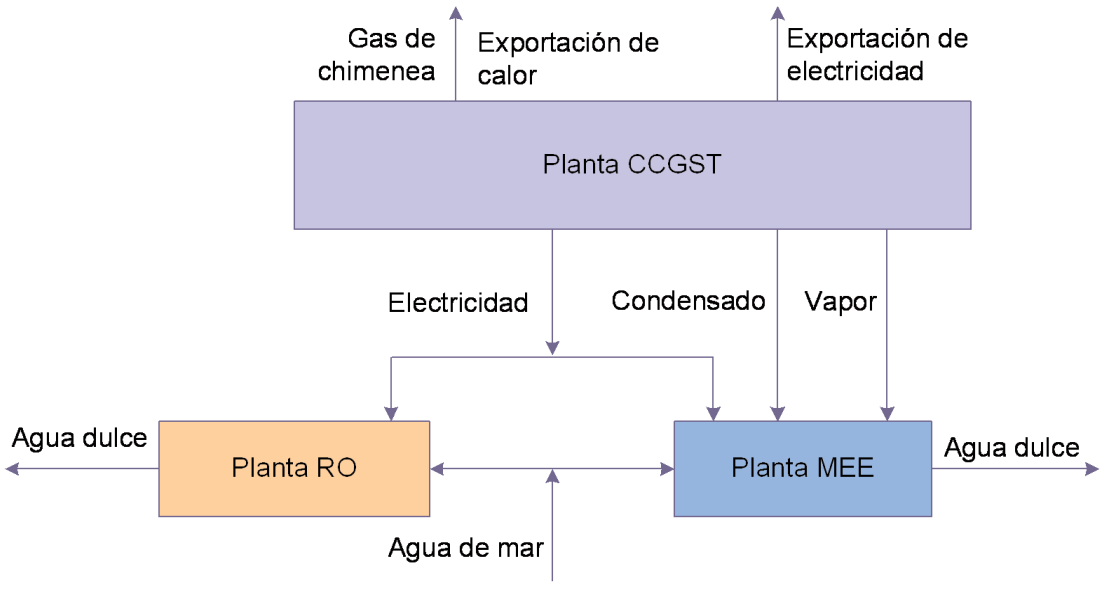

Fuente: Valero, 2001.

Elaboración propia.

\subsection{Comparación de plantas MEE, RO y CDT}

A partir de la década de 1990 la ósmosis inversa se ha convertido en el sistema más difundido, por ser el que presenta un balance económico más favorable. La mayor ventaja es su bajo consumo de energía, que además depende de la salinidad del agua. Sus puntos más conflictivos estriban en el control del agua de entrada y en el mantenimiento de las membranas.

El sistema necesita el suministro estable de energía eléctrica y un control exhaustivo de los parámetros de entrada del agua cruda, mediante un sistema eficaz de pretratamiento del agua.

El sistema de compresión de vapor depende también del suministro fiable de energía eléctrica, y aunque no presenta tantas restricciones en el control del agua de entrada, consume mucha más energía que la ósmosis inversa. 
Estos dos sistemas son más eficaces en cuanto al uso de la energía que los sistemas de destilación (evaporación-condensación), aunque en estos casos la fuente principal sea de origen térmico.

El sistema de evaporación rápida MSF es el más antiguo y se caracteriza por un cierto derroche de energía, requiriendo entre 4 y 5 $\mathrm{kWh} / \mathrm{m}^{3}$ de electricidad para mover todos los equipos auxiliares y un consumo térmico no inferior a los $50 \mathrm{kWh} / \mathrm{m}^{3}$, a un nivel de temperatura entre 100 y $110^{\circ} \mathrm{C}$.

Aproximadamente la mitad de las plantas desaladoras en todo el mundo se han construido por este método, lo que le confiere una cierta predominancia. Sin embargo, su utilidad está limitada a plantas de gran tamaño y a lugares que dispongan de una fuente de energía térmica abundante y barata.

El método de destilación de múltiple efecto (MEE) es la variante moderna del MSF en cuanto a que presenta todas las ventajas de este, así como algunos inconvenientes, como el derroche de energía y la capacidad de construir plantas más pequeñas.

Las plantas MEE requieren un consumo eléctrico de 2 a $3 \mathrm{kWh} / \mathrm{m}^{3}$ y un consumo térmico de 30 a $50 \mathrm{kWh} / \mathrm{m}^{3}$, lo que las acerca a la eficiencia de las plantas de ósmosis inversa (RO), especialmente si el agua por desalinizar es agua de mar.

Los sistemas de destilación solar han demostrado tanto su viabilidad como sus limitaciones. Desde la primera planta construida en el desierto de Atacama, en el norte de Chile, a mediados del siglo XIX, hasta las últimas experiencias llevadas a cabo a finales de siglo XX, los informes hablan de plantas viables, de bajo costo, de reducido mantenimiento, pero de una producción que oscila entre 1 y 4 litros día $/ \mathrm{m}^{2}$, lo que implica unas extensiones muy grandes de superficie o unas producciones demasiado bajas para las expectativas de la comunidad.

Finalmente, es muy importante considerar las tecnologías emergentes, las que han requerido de inversión e investigación significativas; destaca entre ellas la tecnología de desionización capacitiva CDT (CDI). Hasta este momento operan con agua salobre, pero se prevé aplicarlas al agua de mar a partir de este año (2007) (CDT System Inc., 2004; EPRI, 2003; Sabrex of Texas, 2003; Unesco, 2000). 


\section{COSTOS ESTIMADOS DE INVERSIÓN Y PRODUCCIÓN}

Preliminarmente se han analizado las tecnologías recomendables para localidades seleccionadas en la costa del norte del Perú y en el sur de Lima. A continuación se muestran algunos indicadores de evaluación económica (véanse cuadros $6,7,8$ y 9).

\section{Cuadro 6}

Principales indicadores de la evaluación económica de las tecnologías seleccionadas para el distrito de Sechura (Piura)

\begin{tabular}{lcc}
\hline \multicolumn{1}{c}{ Distrito: Sechura } & CDT & RO \\
\hline Capacidad $\left(\mathrm{m}^{3} /\right.$ día) & 30 & 30 \\
Valor de venta $\left(\mathrm{US} \$ / \mathrm{m}^{3}\right)$ & 1 & 1 \\
Costo de producción (US $\left.\$ / \mathrm{m}^{3}\right)$ & & \\
$\quad$ Primeros 10 años & 0,48 & 0,61 \\
$\quad$ Años siguientes & 0,17 & 0,30 \\
Inversión total (US\$) & 59.520 .000 & 59.520 .000 \\
VANE (US\$) & 15.134 .530 & 4.516 .717 \\
TIRE & $13 \%$ & $11 \%$ \\
Periodo de repago (años) & 21 & 14 \\
Mínimo valor de venta (US $\left.\$ / \mathrm{m}^{3}\right)$ & 0,82 & 0,95 \\
\hline
\end{tabular}

Fuente y elaboración propias.

\section{Cuadro 7}

Principales indicadores de la evaluación económica de las tecnologías seleccionadas para los distritos de San José, Pimentel y Santa Rosa (Lambayeque)

\begin{tabular}{lcc}
\hline Distrito: San José, Pimentel, Santa Rosa & CDT & RO \\
\hline Capacidad ( $\left.\mathrm{m}^{3} / \mathrm{día}\right)$ & 10.000 & 10.000 \\
Valor de venta $\left(\mathrm{US} \$ / \mathrm{m}^{3}\right)$ & 1 & 1 \\
Costo de producción $\left(\mathrm{US} \$ / \mathrm{m}^{3}\right)$ & & \\
$\quad$ Primeros 10 años & 0,42 & 0,56 \\
$\quad$ Años siguientes & 0,17 & 0,30 \\
Inversión total (US\$) & 16.340 .000 & 16.340 .000 \\
VANE (US\$) & 7.855 .584 & 4.556 .164 \\
TIRE & $16 \%$ & $14 \%$ \\
Periodo de repago (años) & 10 & 13 \\
Mínimo valor de venta (US $\left.\$ / \mathrm{m}^{3}\right)$ & 0,70 & 0,83 \\
\hline
\end{tabular}

Fuente y elaboración propias. 


\section{Cuadro 8}

Principales indicadores de la evaluación económica de las tecnologías seleccionadas para el distrito de Chimbote (Ancash)

\begin{tabular}{lcccc}
\hline \multicolumn{1}{c}{ Distrito: Chimbote } & CDT & RO & CCGST-MEE & CCGST-RO \\
\hline Capacidad ( $\left.\mathrm{m}^{3} / \mathrm{día}\right)$ & 30.000 & 30.000 & 30.000 & 30.000 \\
Valor de venta $\left(\mathrm{US} \$ / \mathrm{m}^{3}\right)$ & 1 & 1 & 1 & 1 \\
Costo de producción $\left(\mathrm{US} \$ / \mathrm{m}^{3}\right)$ & & & & \\
$\quad$ Primeros 10 años & 0,48 & 0,61 & & \\
$\quad$ Años siguientes & 0.17 & 0,30 & & 103.740 .000 \\
Inversión total (US $\$$ ) & 59.520 .000 & 59.520 .000 & 108.840 .000 & 19.01 .077 \\
VANE (US $\$$ ) & 15.134 .530 & 4.516 .717 & 73.001 .722 & 76.801 .07 \\
TIRE & $13 \%$ & $11 \%$ & $18 \%$ & $19 \%$ \\
Periodo de repago (años) & 21 & 14 & 9 & 8 \\
Mínimo valor de venta (US $\left.\$ / \mathrm{m}^{3}\right)$ & 0,82 & 0,95 & & \\
\hline
\end{tabular}

Fuente y elaboración propias.

\section{Cuadro 9}

Principales indicadores de la evaluación económica de las tecnologías seleccionadas para los distritos de San Bartolo y Asia (Lima)

\begin{tabular}{lcc}
\hline \multicolumn{1}{c}{ Distrito: Chimbote } & CDT & RO \\
\hline Capacidad ( $\left.\mathrm{m}^{3} / \mathrm{día}\right)$ & 2.000 & 2.000 \\
Valor de venta (US $\left.\$ / \mathrm{m}^{3}\right)$ & 1 & 1 \\
Costo de producción $\left(\mathrm{US} \$ / \mathrm{m}^{3}\right)$ & & \\
$\quad$ Primeros 10 años & 0,36 & 0,50 \\
$\quad$ Años siguientes & 0,17 & 0,30 \\
Inversión total (US\$) & 2.460 .000 & 2.460 .000 \\
VANE (US\$) & 2.194 .663 & 1.534 .779 \\
TIRE & $21 \%$ & $18 \%$ \\
Periodo de repago (años) & 7 & 9 \\
Mínimo valor de venta (US $\left.\$ \mathrm{~m}^{3}\right)$ & 0,56 & 0,70 \\
\hline
\end{tabular}

Fuente y elaboración propias.

\section{CONCLUSIONES}

\subsection{Conclusiones generales}

- En las cuencas hidrológicas de la costa norte peruana y del sur de Lima hay déficit de agua para uso agrario e industrial por más de 50 millones de $\mathrm{m}^{3}$, con excepción de las cuencas de los ríos Tumbes, Chira y Piura. 
- Una causa del déficit de agua es la falta de buenas prácticas de uso del recurso hídrico, en parte originado por las tarifas nominales que se aplican al servicio de suministro a escala nacional.

- No debe haber competencia entre la producción de agua alternativa y la producción de agua convencional. Por el contrario, estos dos bienes deben ser complementarios, dado el déficit existente en el suministro de agua convencional.

- En el mundo se están desarrollando nuevos procesos para desalar agua marina, mejorando la superficie de transferencia de calor en las plantas actuales y aplicando la desionización capacitiva como una nueva tecnología alternativa.

- El mercado para la desalación de agua dependerá de los costos del agua producida en las plantas desaladoras y del impacto de sus emisiones hacia el ambiente.

- Las tecnologías de desalación por ósmosis inversa y por desionización capacitiva son las más aplicables a las localidades consideradas en este artículo.

- La adaptación de las mencionadas tecnologías de desalación a ciclos de cogeneración permite obtener costos de producción más bajos.

- La tecnología desaladora por separación eléctrica ha empezado a competir con las tecnologías de desalación por membrana y desalación térmica, con una gran perspectiva de éxito.

- El impacto ambiental derivado de la instalación de una planta desaladora comprende el vertido de salmueras, efluentes, emisiones y ruidos. Su naturaleza es comparable con los impactos de las industrias de procesos químicos en general.

\subsection{Conclusiones técnicas y económicas}

- La tecnología de desionización capacitiva (CDT) es la que arroja los mejores indicadores económicos, seguida por las de ósmosis inversa (RO) y evaporación con múltiples efectos (MEE), debido a que desala agua salobre. El costo de producción unitario del agua desalada obtenida por CDT a partir del décimo año es de $0,17 \mathrm{US} \$ / \mathrm{m}^{3}, 45 \%$ menor que su similar en ósmosis inversa. 
- A igual capacidad de producción los costos de inversión son diferentes, debido principalmente a la complejidad del proceso. La tecnología de evaporación rápida con múltiples etapas (MSF) es la más costosa, con $33 \%$ más que su similar en desionización capacitiva.

- Para que estos proyectos sean factibles de ser financiados, se requiere que la depreciación de los activos sea mayor de 10 años, que las tasas de interés sean menores de $10 \%$, que los periodos de amortización sean mayores de 5 años y que los periodos de evaluación sean de 15 a 30 años, teniendo en cuenta que la inversión se recupera en más de 10 años. Estas cifras no son muy alentadoras para el inversionista privado, lo que hace necesaria la intervención del Estado y de fuentes de financiamiento internacionales (CAF, BID).

- Las tecnologías alternativas propuestas en este artículo arrojan indicadores económicos más alentadores que sus similares por separado; a pesar de tener costos de inversión mayores.

- Los factores que hacen que dichos indicadores económicos sean mejores son dos: la venta del excedente de energía eléctrica y el abaratamiento de los costos de energía debido al efecto de la cogeneración.

- De las tres tecnologías alternativas estudiadas, la tecnología híbrida CCGST-RO-MEE es la que posee la tasa interna de retorno más alta y una menor sensibilidad ante posibles variaciones del valor de venta de los productos (agua desalada y energía eléctrica).

- La desionización capacitiva resulta ser la más apropiada para los distritos de Sechura (30.000 m³/día); San José, Pimentel y Santa Rosa (10.000 m³/día), y San Bartolo y Asia (2.000 m³/día).

- Tanto la planta dual de evaporación con múltiples efectos y ciclo combinado con turbinas de gas y de vapor (CCGST-MEE) como la planta dual de ósmosis inversa y ciclo combinado con turbinas de gas y de vapor (CCGST-RO) son las más apropiadas para el distrito de Chimbote (30.000 m²/día).

- Las tecnologías híbridas y duales que se proponen son las más atractivas para el inversionista privado, por ser las que ofrecen un periodo de repago menor de 10 años. 


\section{BIBLIOGRAFÍA}

American Membrane Technology Association. How much does desalted water cost?, 2003 [en línea] .<http://www.membraneamta. org/media/pdf/desaltingcost.pdf.> (Consulta: 23 de diciembre del 2003).

BBC, Inc. Aerogel process nears market. Norwalk, Connecticut, 2004 [en línea]. <http://www.cdwater.com/press/04_01H2Onews.php> (Consulta: 3 de agosto del 2004).

California Coastal Commission. Seawater desalination in California. California, 1998. [en línea]. <http://www.coastal.ca.gov/ desalrpt/dchap3.html> (Consulta: 26 de setiembre del 2004).

California Water Desalination Task Force. Desalination technology working paper. California, 2000 [en línea]. <http://www.owue. water.ca.gov/recycle/desal/Docs/DesalTechnology.doc> (Consulta: 26 de setiembre del 2004).

CDT Systems, Inc. Capacitive DeionizationTechnology. Texas, 2004 [en línea]. <http://www.cdtwater.com/water.php.> (Consulta: 4 de julio del 2004).

—. CDT Mobile Systems. Texas, 2004 [en línea]. <http://www.cdtwater.com/water.php.> (Consulta: 4 de julio del 2004).

Electric Power Research Institute (EPRI). Emerging environmental technologies. California: California Energy Commission, 2003 [en línea] <http://www.energy.ca.gov/reports/2004-01-22_50003-068C.PDF> (Consulta: 26 de setiembre del 2004).

Ettouney, H. M. y H. T. El-Dessouky. Evaluating the Economics of Desalination. Kuwait: Kuwait University, 2002 [en línea]. $<$ http://www.cepmagazine.org/pdf/120232.pdf> (Consulta: 26 de setiembre del 2004).

Fariñas, M. Ósmosis inversa: Fundamentos, tecnología y aplicaciones. Nueva York: McGraw-Hill, 1999.

ILCE. La hidrósfera: Desalinización del agua del mar. México, 1985 [en línea]. <http://lectura.ilce.edu.mx:3000/biblioteca/sites/ciencia/volumen2/ciencia3/097/htm/sec_10.htm> (Consulta: 20 de diciembre del 2003). 
Instituto Nacional de Recursos Naturales (Inrena). Mapas del Perú Ambiental. Lima, 2004 [en línea]. <http://www.minag.gob.pe/ rrnn_a_est.shtml> (Consulta: 23 de diciembre del 2003).

Lawrence Livermore National Laboratory (LLNL). Carbon aerogel deionization of water, 2003. [en línea]. <http://www. llnl.gov/IPandC/technology/profile/aerogel/CarbonAerogelCapacitiveDeionizationOfWater/index.php> (Consulta: 10 de enero del 2004).

Miller, J. E. Review of water resources and desalination technologies. Albuquerque, Nuevo México: Sandia National Laboratories, 2003 [en línea]. <http://www.cepmagazine.org/pdf/120232.pdf> (Consulta: 26 de setiembre del 2004).

Ministerio de Agricultura. Hidrometeorología. Lima, 2003 [en línea]. $<$ http://www.portalagrario.gob.pe/hidro_drenaje_imp.shtml> (Consulta: 23 de diciembre del 2003).

Ministerio de Energía y Minas, Dirección de Hidrocarburos. Ventajas del uso del gas natural en la industria. Lima, 2003 [en línea]. .<http://www.minem.gob.pe> (Consulta: 5 de enero del 2004).

Moss, E. G.; Hunt, E. T. y C. A. Kiefer. Comparison of multiple effect distillation and reverse osmosis for New Providence Island. Florida: Stone \& Webster Eng. Co., 1994 [en línea]. <http:// www.desalco.ky./d-htlm/papers.htm> (Consulta: 23 de diciembre del 2003).

Sabrex of Texas, Inc. EWP Technology. Texas, 2003. [en línea]. $<$ http://www.sabrexewp.com> (Consulta: 3 de setiembre del 2004).

—. EWP Test report of Electronic Water Purifier. Texas, 2003 [en línea]. <http://www.sabrexewp.com> (Consulta: 18 de julio del 2004).

- Removal of chrome and creating a zero discharge system using Electronic Water Purification. Texas, 2003 [en línea]. $<$ http://www.sabrexewp.com> (Consulta: 18 de julio del 2004).

Unesco-IHE. Water Scarcity and desalination. Francia, 2000 [en línea]. <http://www.ihe.nl/vmp/articles/News/NEW-JAN-01-BG02.html> (Consulta: 23 de diciembre del 2003). 
Universidad de Alicante. Desalar el agua a bajo costo, una batalla para la ciencia. España, 2003 [en línea]. <http://www.ua.es/es/ servicios/comunicacion/notas/03042301.html> (Consulta: 21 de diciembre del 2003).

Valero, A.; Uche, J. y Serral. La desalación como alternativa al Plan Hidrológico Nacional. Zaragoza: Universidad de Zaragoza, 2001 [en línea]. <http://www.cirse.cps.unizar.es/> (Consulta: 26 de setiembre del 2004). 